\title{
TRAJECTORY BASED 3D FRAGMENT TRACKING IN HYPERVELOCITY IMPACT EXPERIMENTS
}

\author{
E. Watson ${ }^{1 *}$, H.-G. Maas ${ }^{2}$, F. Schäfer ${ }^{1}$, S. Hiermaier ${ }^{1}$ \\ ${ }^{1}$ Fraunhofer Institute for High-Speed Dynamics, Ernst Mach-Institut, EMI, 79104 Freiburg, Germany \\ ${ }^{2}$ Technische Universitt Dresden, Institute of Photogrammetry and Remote Sensing, 01062 Dresden, Germany
}

Commission II, WG II/7

KEY WORDS: Hypervelocity impact, Space debris, Fragmentation, Particle tracking, Trajectory fitting, Stereo matching

\begin{abstract}
:
Collisions between space debris and satellites in Earth's orbits are not only catastrophic to the satellite, but also create thousands of new fragments, exacerbating the space debris problem. One challenge in understanding the space debris environment is the lack of data on fragmentation and breakup caused by hypervelocity impacts. In this paper, we present an experimental measurement technique capable of recording 3D position and velocity data of fragments produced by hypervelocity impact experiments in the lab. The experimental setup uses stereo high-speed cameras to record debris fragments generated by a hypervelocity impact. Fragments are identified and tracked by searching along trajectory lines and outliers are filtered in 4D space (3D + time) with RANSAC. The method is demonstrated on a hypervelocity impact experiment at $3.2 \mathrm{~km} / \mathrm{s}$ and fragment velocities and positions are measured. The results demonstrate that the method is very robust in its ability to identify and track fragments from the low resolution and noisy images typical of high-speed recording.
\end{abstract}

\section{INTRODUCTION}

Space debris poses a major danger to continued human operations in orbits around the earth (Liou, 2006). In Low Earth Orbit, satellites and space debris circle the earth at over $7 \mathrm{~km} / \mathrm{s}$ and any collisions at such speeds, known as hypervelocity impacts (HVI), can be extremely destructive. Not only is there the danger of catastrophic failure for the satellite, but HVI events also create thousands of new debris that further exacerbate the space debris problem. To mitigate and understand the risks to operational satellites, researchers use debris environment models to model the debris orbiting Earth (Klinkrad et al., 2001, Krisko, 2010). These models reliably predict the orbits of large fragments $(>10 \mathrm{~cm})$ using ground based sensors, but show discrepancies in modeling smaller but still dangerous fragments in the critical range (1 mm to $1 \mathrm{~cm})$ which cannot be directly measured from Earth (Krisko et al., 2015). This discrepancy highlights the need for a more thorough understanding of HVI and fragmentation phenomena for modeling of the space debris environment.

The lower limit of the hypervelocity regime is more appropriately defined by the onset of specific impact phenomena, rather than any absolute minimum velocity. The most general criteria is the complete pulverization of the impactor and target in the immediate vicinity of the impact (Swift, 1982). The lower limit of this velocity varies widely depending on the material's shock impedances and can be anywhere between 2 and $10 \mathrm{~km} / \mathrm{s}$ (Zukas et al., 1982). Upon impact, strong shockwaves travel through the projectile and the target. Upon reaching free surfaces, the shockwave is reflected as a rarefaction, or tensile, wave, releasing the compressed material and causing fragmentation, melting, and vaporization.

HVI experiments are typically performed with two-stage lightgas guns (Putzar and Schaefer, 2009), which are able to acceler-

\footnotetext{
*Corresponding author: erkai.watson@emi.fraunhofer.de
}

ate projectiles up to $9 \mathrm{~km} / \mathrm{s}$. The simplest experiments involve the impact of a spherical projectile with a thin plate. Upon impact, the sphere and plate fragment into a cloud of debris. High-speed video imaging of the developing debris cloud, like that shown in Fig. 1, is the most common tool for studying HVI fragmentation (Zhang et al., 2008, Piekutowski, 1997). This method allows for a qualitative understanding of the fragmentation process, but cannot measure quantitative data on individual fragments of the debris cloud. The high-speed images are often supplemented by the use of a witness plates or soft catcher which can provide insight into the composition of the fragments and the spray angle of the debris (Higashide et al., 2015, Nishida et al., 2013), but still provide little time resolved data.

Measuring particle velocities in HVI experiments has been previously investigated with techniques based on Particle Image Velocimetry (PIV) and Particle Tracking Velocimetry (PTV), two non-intrusive imaging techniques originally developed for experimental fluid dynamics. PIV works with interrogation areas and uses convolution to find the motion vector of the areas between frames (Raffel et al., 2007). In the realm of HVI, 3D PIV has been used to study crater formation (Heineck et al., 2002). Such techniques are able to measure the flow-field of the ejected particles, but are restrict to areas and volumes and are unable to provide individual fragment data.

Alternately, PTV identifies and tracks individual particles (Maas et al., 1993) and is therefore more suited to the study of HVI fragmentation. A combined 2D PIV/PTV technique has been used to study HVI crater growth in sand, allowing the velocities and position of a small subset of sand grains to be measured (Hermalyn and Schultz, 2011). In a later paper, the method was extended to $3 \mathrm{D}$ using four cameras to find the 3D locations of individual sand particles and using PIV to help link particles between two frames to find their velocities (Hermalyn et al., 2014). In other research, a pure particle tracking technique has been applied to HVI experiments in geological materials in $2 \mathrm{D}$, where both velocities 

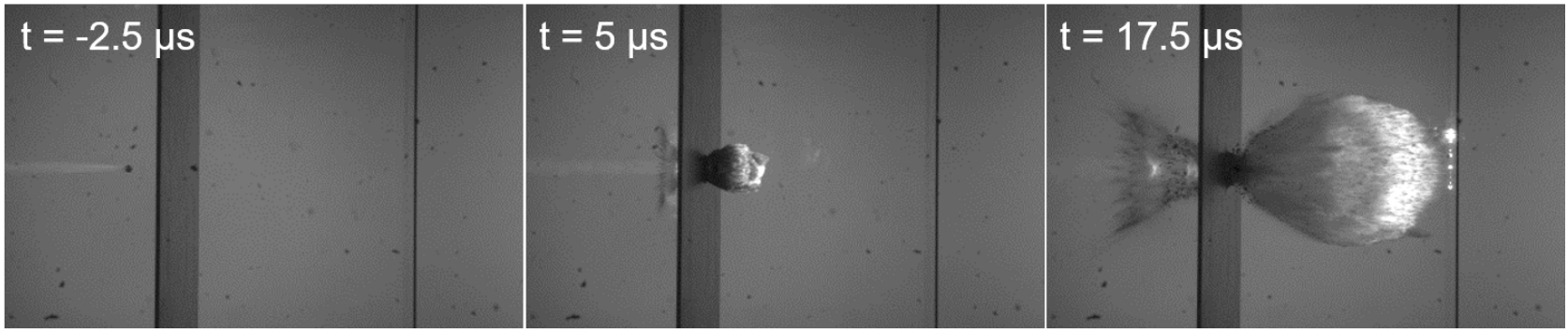

Figure 1. Hypervelocity impact experiment performed at Fraunhofer EMI's two-stage light-gas gun facility. The spherical projectile, travleing at $6.8 \mathrm{~km} / \mathrm{s}$ impacts the target plate from left to right. A cloud of debris fragments is generated and impacts the witness plate on the right.

and sizes of the ejected fragments were determined (Gulde et al., 2017, Schimmerohn et al., 2018). In these studies, particle tracking was aided by taking advantage of the constant velocities of the ejected fragments to help filter out outliers. A similar 2D algorithm was applied to the study of debris cloud formations in HVI of satellite shields (Watson et al., 2017).

In this paper, we present a 3D particle tracking technique for analyzing the debris cloud generated by HVI experiments. Our goal is to extract quantitative data on individual debris fragments, such as $3 \mathrm{D}$ position and velocity from these experiments. We improve on previous work, in particular the correspondence problem of identifying the same image in both cameras, by limiting our search to fragment trajectories. Our results show that the method is able to robustly measure fragment data from the low resolution and noisy images typical of high-speed images.

\section{SETUP}

\subsection{Experimental Setup}

The HVI experimental setup is shown in Fig. 2. The setup uses a two-stage light-gas gun to accelerate an aluminum projectile to between 3 and $9 \mathrm{~km} / \mathrm{s}$. The target consists of an aluminum target plate followed by a copper witness plate placed in a vacuum chamber. The HVI causes the projectile and parts of the target plate to fragment into a cloud of debris, which continue on at high speed to crater the witness plate. A continuous-wave laser volume, placed in the path of the expanding debris cloud, illuminates the fragments. A pair of high-speed video cameras record the experiment.
In this paper, we focus our attention on one experiment performed at our light-gas gun facility at Fraunhofer EMI and use it to demonstrate the fragment tracking algorithm. In this experiment, a 7 $\mathrm{mm}$ diameter aluminum sphere was accelerated to $3.2 \mathrm{~km} / \mathrm{s}$ at Fraunhofer EMI's two-stage light-gas gun facility. We chose a low impact velocity to maximize the projectile fragmentation while minimizing melting and vaporization of the fragments (Piekutowski, 2003). The target was a $1.5 \mathrm{~mm}$ thick aluminum bumper plate followed by a copper witness plate with a $199 \mathrm{~mm}$ standoff distance. The impact occurred in a 0.1 mbar vacuum. Experiment parameters are summarized in Table 1.

\begin{tabular}{|l|l|}
\hline \multicolumn{2}{|c|}{ Experimental Parameters (\#5780) } \\
\hline Projectile & $7 \mathrm{~mm} \mathrm{A199}$ sphere \\
Target & $1.5 \mathrm{~mm}$ Al99 plate \\
Witness Plate Standoff & $199 \mathrm{~mm}$ \\
Impact Velocity & $3.2 \mathrm{~km} / \mathrm{s}$ \\
Target Chamber Pressure & $0.1 \mathrm{mbar}$ \\
Laser Type & $18 \mathrm{~W} \mathrm{CW}$ at $532 \mathrm{~nm}$ \\
\hline
\end{tabular}

Table 1. Experimental parameters

\subsection{Calibration and Coordinate System}

Our measurement system uses two synchronized high-speed CMOS cameras with micro- to nanosecond exposure times, capable of up to 10 million frames per second, to capture the fragment trajectories. The camera parameters are summarized in Table 2. Prior to the HVI experiment, we calibrate our stereo camera system with a $2 \mathrm{D}$ reference pattern. Twenty-four image pairs of the $2 \mathrm{D}$ pattern, shown in Fig. 3, are used, allowing us to find the intrinsic
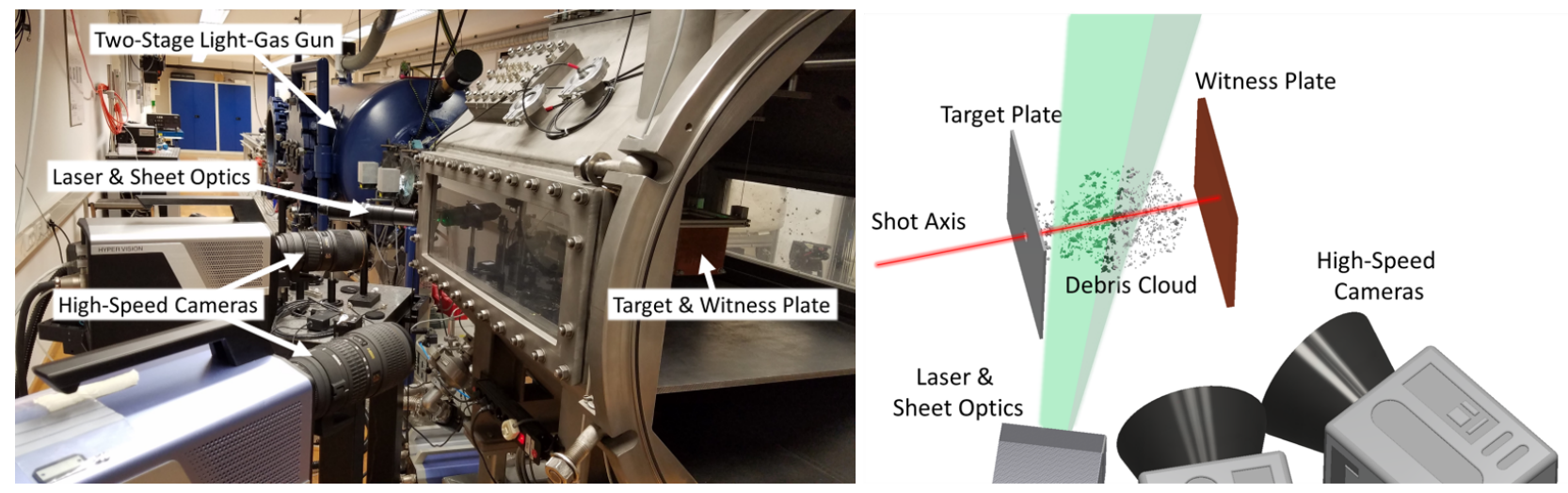

Figure 2. Setup for hypervelocity impact experiment to measure 3D spatially and temporally resolved fragmentation data. The experiment involves using two synchronized high-speed cameras to record the flight of debris fragments following a HVI event. The fragments are illuminated as they pass through the laser volume. 


\begin{tabular}{|l|l|l|}
\hline \multicolumn{3}{|c|}{ Camera Parameters } \\
\hline & Left Camera & Right Camera \\
Camera Model & Shimadzu HPV-X2 & Shimadzu HPV-X \\
Field of View & $56 \times 35 \mathrm{~mm}$ & $72 \times 45 \mathrm{~mm}$ \\
Focal Length & $200 \mathrm{~mm}$ & $200 \mathrm{~mm}$ \\
Aperture & $\mathrm{f} / 8^{*}$ & $\mathrm{f} / 2.8$ \\
\hline Framerate & 1.67 million fps $(40$ frames over $24 \mu \mathrm{s})$ \\
Exposure Time & \multicolumn{2}{|c}{$270 \mathrm{~ns}$} \\
Image Size & \multicolumn{2}{|c}{$400 \times 250$ pixels } \\
\hline
\end{tabular}

Table 2. Camera parameters used in the experiment. *approximate value, aperture not labeled

and extrinsic camera parameters (Zhengyou Zhang, 1999). Because of small field of view (large focal length) of our cameras, we assume the principal point of each camera to be at the center of the image (Ruiz et al., 2002). Radial distortion is accounted for with a first order function.

The world coordinate system used in the analysis is defined with its origin at the left camera. A crucial part of the analysis relies on being able to reconstruct the relative locations of the target and witness plates, shot axis, and fragment cloud in this world coordinate system. Since the target and witness plates are not within the field of view of the cameras, and the shot axis is not visible, we determine these coordinates in a separate step.

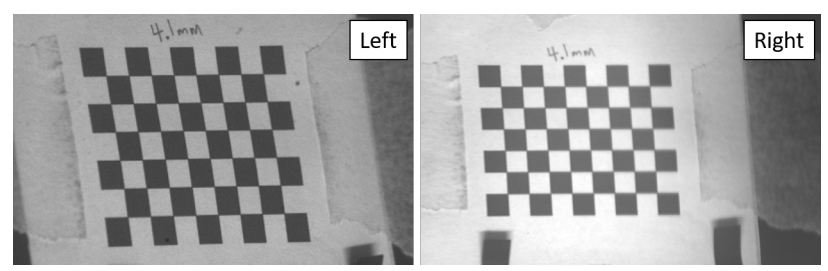

Figure 3. 2D calibration target used for finding the intrinsic and extrinsic camera parameters

After the experiment, we determine the location of the shot axis by using a laser, shown in red in Fig. 2. We visualize points along the laser beam with a semi transparent cross-hairs target, shown in Fig. 4. The cross-hairs are places in the laser's path and its location is recorded with the cameras. Four or five points are recoded and the best-fit line is taken as the shot axis.

Once the shot axis is known, the positions of the witness and target plates can be determined by measuring the distance along the shot axis to the witness and target plates. The result is the complete geometry defined in world coordinates.
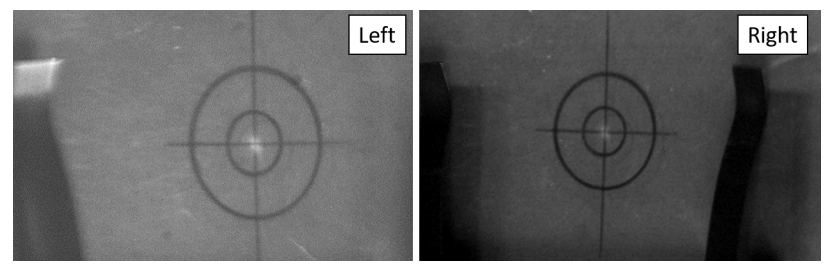

Figure 4. Semi-transparent cross-hairs used to define the experiment shot axis in world coordinates. A laser shinning along the shot axis is used to position the cross-hairs. Multiple points are measured to define the line.

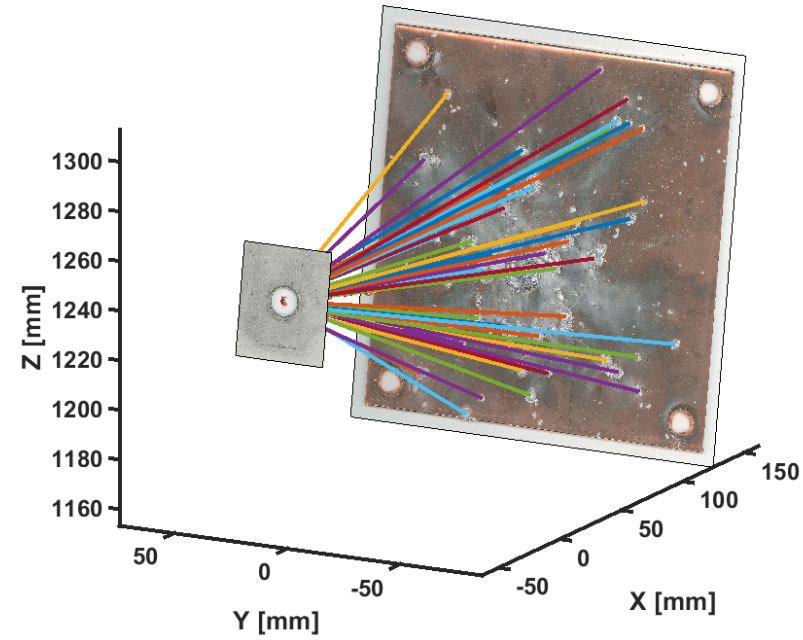

Figure 5. The target, witness plate, and shot axis in world coordinates. Craters in the witness plate and the location of the target hole are used to estimate the 3D trajectories of each fragment. Shown here are the best fitting trajectories for each crater.

\section{METHODS}

Although a considerable amount of research has been conducted on stereo measurements and particle tracking, many aspects of past work cannot be directly applied to HVI measurement images because of limitations specific to high-speed measurements. For example, in the case of this study, the availability of high-speed cameras capable of millions of frames per second limits the number of cameras to the bare minimum needed for stereo vision. This, combined with the low resolution and noisy images, makes it impractical to reliably find corresponding fragments with the standard PTV approach of identifying fragment in each image and using epipolar geometry to match correspondences (Maas et al., 1993). Similarly, trajectory linking techniques that rely on feature detection or a cost function minimization (Ouellette et al., 2006) suffer from either low spatial or temporal resolution respectively.

Despite these limitations, the unique physics of the experiment lend themselves to some simplifying assumptions which allow for robust identification and trajectory linking algorithms. The first assumption we make is that each fragment travels in a straight line, since gravitational forces are negligible because of the very short experiment time scale, on the order of micro-seconds. Second, we assume fragments travel at constant velocity, since there is little air resistance acting in the evacuated target chamber.

Figure 7 shows a flowchart of the algorithm. The main building block of the fragment tracking algorithm is the trajectory. Straight line trajectories are estimated and projected into the highspeed images to find the fragment that belongs to that trajectory. Trajectories are ranked and the best ones are deemed to contain the fragments seen in the images.

The first step involves determining the world coordinates of the craters in the witness plate and looping over each crater. These craters are one end of the trajectory in world coordinates. Next, the launch locations on the target plate needs to be defined. We assume that each fragment's launch location on the target plate 

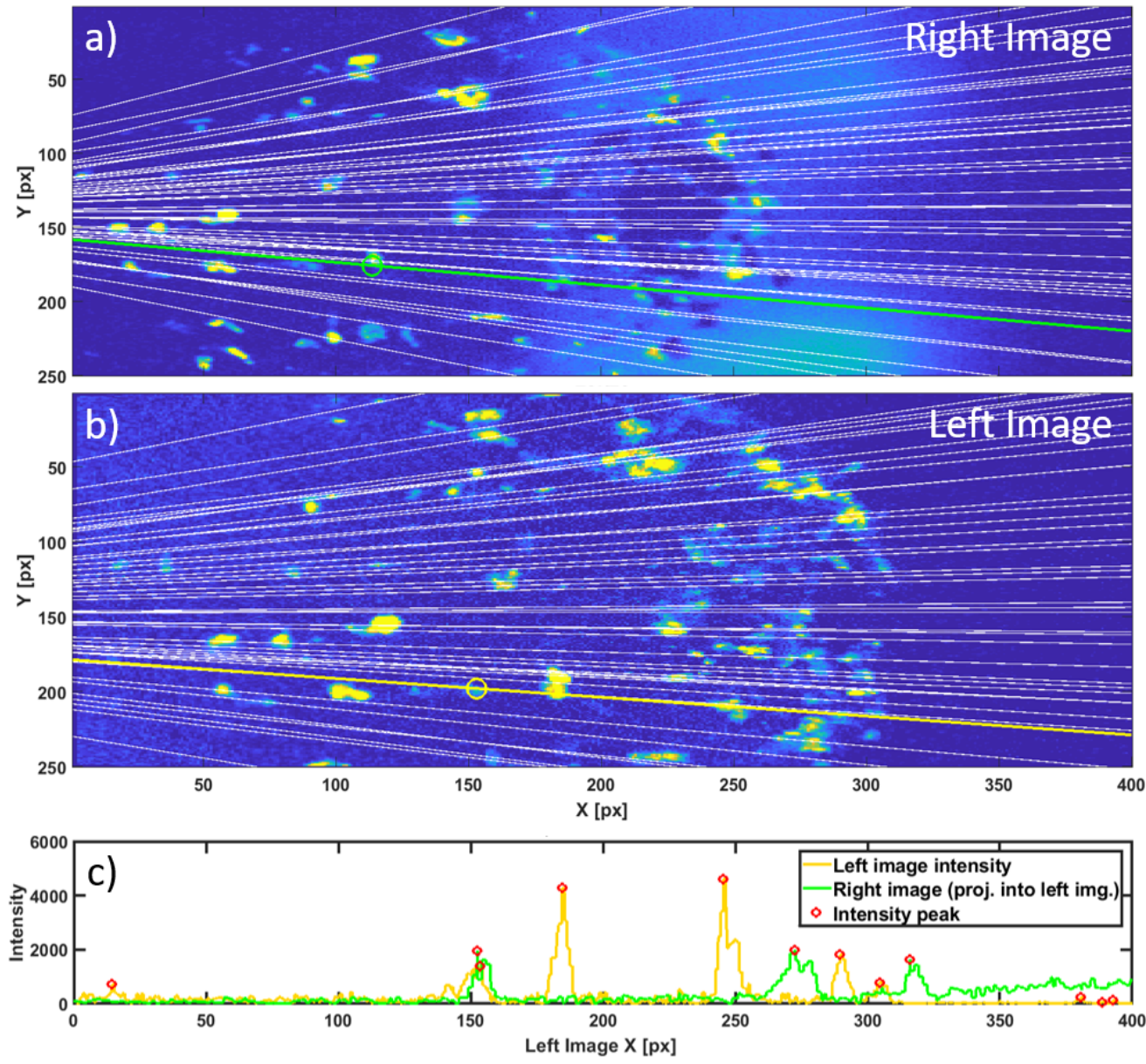

Figure 6. 3D trajectories shown in Fig. 5 are projected into the right (a) and left (b) camera images and shown as white lines. One trajectory is plotted as a yellow line in the left image (b). The pixel intensities along that trajectory are plotted in (c) as a yellow line. The same trajectory is plotted in green in the right image (a). Via epipolar geometry, intensities in the right image are plotted at their corresponding left image pixel location, allowing a direct comparison of intensities between left and right images (along the same 3D trajectory). Converging peaks represent the same fragment on the trajectory.

follows the pattern found on the witness plate, i.e. if the crater is on the right side of the witness plate, then the launch location is also on the right side of the target hole. Another way of expressing this is that the trajectories move in a radially expanding pattern and generally do not cross one another (Piekutowski, 1993). Since the launch location is only approximate, for every crater, we vary the assumed launch location. This creates a double loop of craters and launch locations each defining a trajectory. Figure 5 shows the target and witness plates along with a set of estimated trajectories.

For each trajectory, we perform the following steps. The 3D trajectory is projected into each $2 \mathrm{D}$ camera image by multiplying the line's end points with each camera's parameter matrix $P$. Since this 2D line is the fragment trajectory, we expect to find a fragment in both the right and left images that moves along this line. This reduces our search to a $1 \mathrm{D}$ problem. The fragment trajectories shown in Fig. 5 are projected into the 2D camera images as white lines shown in Fig. 6. Next, we search along these trajectories for the unique matching fragments.

On each trajectory in the $2 \mathrm{D}$ left and right images, there may be multiple fragments, but only the pair that triangulates to a point on the 3D trajectory is the correct one. To find the pair, we es- tablish a left and right image pixel to pixel correspondence, along each trajectory, by finding the intersection of each pixels epipolar line with the trajectory. This correspondence allows us to match the intensities in the two images along that trajectory. The point of convergence of two intensity peaks then represents a fragment on that given trajectory. This matching is illustrated in Fig. 6 where one trajectory is highlighted in the left and right images. Figure $6 \mathrm{c}$ plots the 1D pixel intensities against the left image $\mathrm{x}$ coordinate in yellow and also projects the intensity values of the right image into the left image $\mathrm{x}$-pixel coordinates in green. We see that the point where both peaks converge $(x=150)$ corresponds to the location of the fragment in the left image. This location is marked with a circle in each image. Given the coordinates of this point in the left and right images, we triangulate to find that its world coordinates do indeed lie on the 3D trajectory line.

In practice, there are many instances when it is not clear which pair of 1D peaks (Fig. 6c) truly corresponds to the fragment on the $3 \mathrm{D}$ trajectory. To overcome this ambiguity, we do not attempt to determine the correct pair of points from Fig. $6 \mathrm{c}$, but rather take every combination of pairs and determine their world coordinates. Collecting all these points over the entire image sequence yields a cloud of points in world coordinates. We filter out the bad $3 \mathrm{D}$ points by defining a maximum distance from the $3 \mathrm{D}$ tra- 


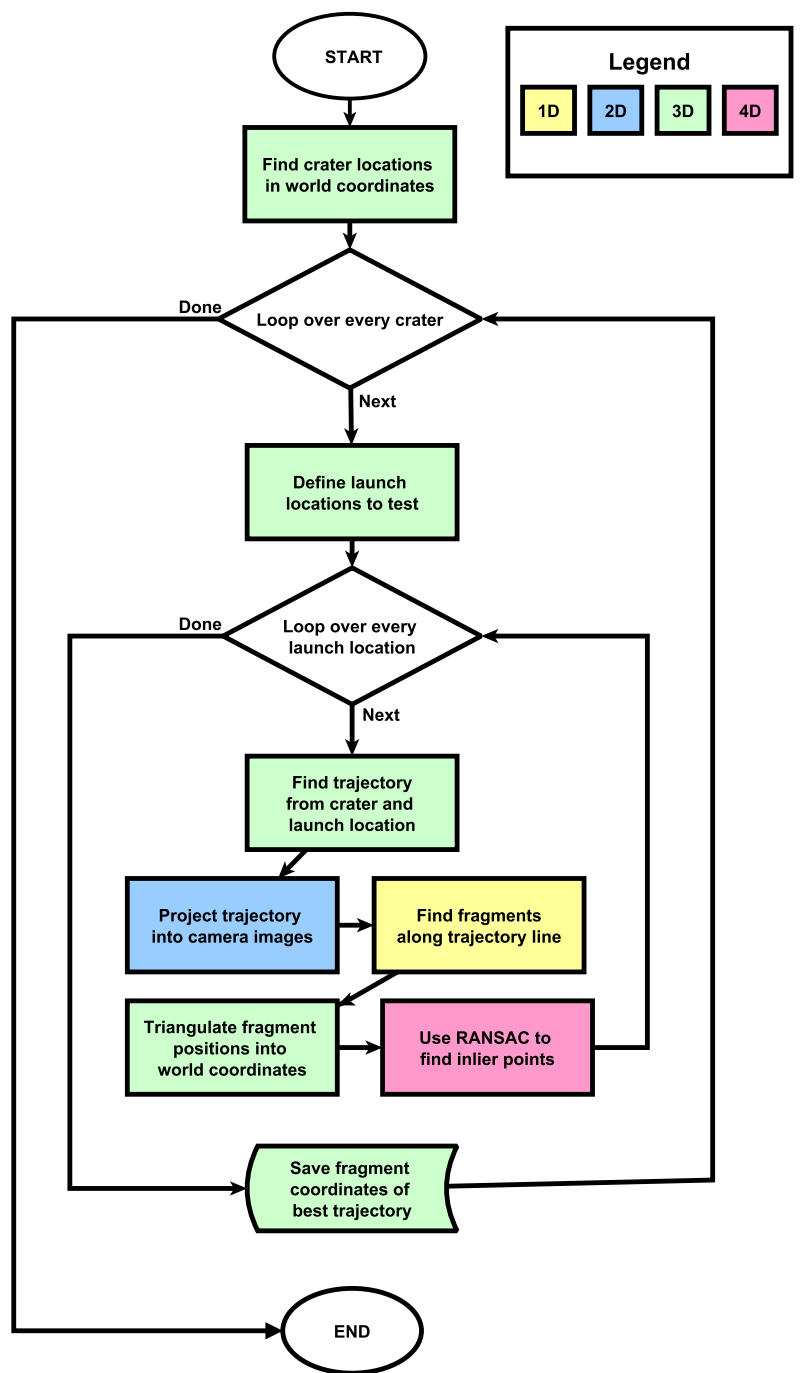

Figure 7. Flowchart of algorithm for identifying fragments based on a trajectory search method.

jectory line, $r_{\max }$; only points within this distance are considered inliers. After filtering, the remaining points are all on the trajectory, but have not yet been filtered by time. Since we assume constant velocity motion, we expect the trajectory to be a straight line in 4D space (3D position + time). We apply a random consensus algorithm (RANSAC) (Fischler and Bolles, 1981) in this 4D XYZT space to identify the subset of points that fit the trajectory and have a constant velocity. A simplified figure with the $\mathrm{Z}$ dimension omitted is shown in Fig. 8. In this figure, the blue dots represent all pairs of 1D peaks found over the entire time sequence. Green points are those that lie within the distance $r_{\max }$. These green points are the inputs to the RANSAC algorthim. The outputs are the red dots which represent the actual locations of the fragment in world coordinates as it moves along its trajectory.

As pictured in Fig. 7, this analysis is performed for every crater and every launch location. From each group of tested trajectories (all belonging to one crater), we choose the trajectory with most identified fragments. This process of testing slight trajectory variations, ensures that slight errors in defining the target ,witness plate, and shot axis locations do not prevent us from finding the fragments belonging to each trajectory. Finally, once the best trajectory for each crater is chosen, we save the identified fragment

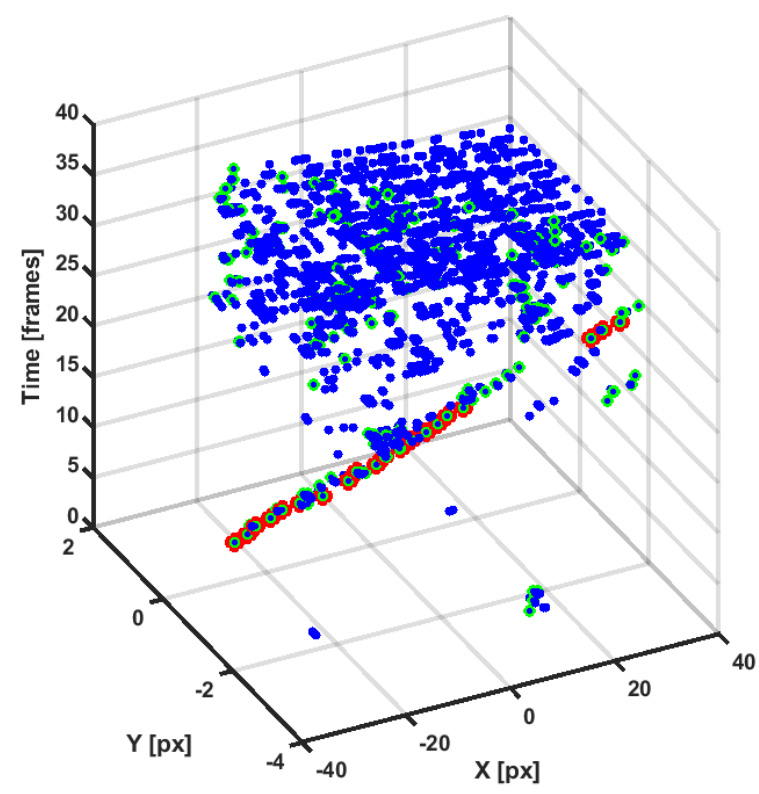

Figure 8. Peak pairs from the 1D intensity analysis of Fig. 6 over all time frames are triangulated to yield a cloud of points. These points (blue dots) are sorted into 4D space (3D + time). Here the $\mathrm{Z}$ component is omitted. Points within a distance $r_{\max }$ from the trajectory are shown in green. Points near the trajectory that are also consistent in time are found with RANSAC and plotted in red. Red points are the true fragment coordinates.

locations.

\section{RESULTS}

The output of the algorithm presented in the previous section is a list of world coordinate positions and times of the fragments that created the craters in the witness plate. In this particular experiment, 45 craters have been identified in the copper witness plate. From the 45 craters, we tracked 37 corresponding fragment trajectories in the high-speed images, or a $82 \%$ success rate. The length of these trajectories, i.e. the number of frames over which the fragment was tracked, averaged 20 frames. Figure 9 shows a histogram of the trajectory length for the 37 tracked fragments.

Apart from the 3D locations of the tracked fragments, the velocity of each fragment was also calculated and is shown in Fig. 10. The red line in this figure indicates the impact velocity of the aluminum projectile. As expected, the velocity of the fastest fragments is only slightly below that of the impact velocity (Piekutowski, 1997).

Although the original aim was to record the fragments illuminated in the laser volume, we found that it was also possible to identify and track fragments both before they entered, and after they left the laser volume, when they were only illuminated by scattered laser light. This was possible because the algorithm is very insensitive to noisy images and even faintly lit fragments can be recognized. 


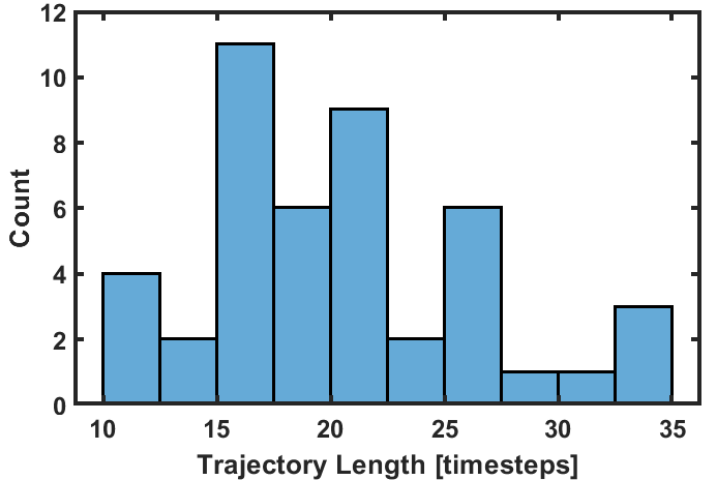

Figure 9. Histogram of trajectory length, defined as the number of frames where the fragment belonging to the trajectory was identified. Since the image sequence was 40 frames, some fragments were tracked over nearly the entire length of the recording.

\section{MEASUREMENT ACCURACIES}

We determine the measurement accuracies of the experimental setup based on the fragments that were identified as part of a trajectory. We reiterate our assumption that fragments travel in straight lines and move with constant velocity. Given the calculated velocity of each fragment and the frame to frame time, we define an expected position of the fragment in the next image. The difference between the expected position and the actual fragment position is defined as the position error. The position error from the 37 trajectories, representing 697 samples, is shown in Fig. 11.

We define a tolerance interval for the position measurement where the upper and lower bounds are defined as

$$
\begin{aligned}
& X_{U}=\mu+k s \\
& X_{L}=\mu-k s,
\end{aligned}
$$

where $\mu$ is the mean and $s$ is the standard deviation of the sample, and $k$ is the two-sided tolerance factor. The constant $k$ is a function of $p$, a proportion of the sample population, and $\gamma$, the confidence. For our tolerance interval we define $p=0.90$ and $\gamma=0.99$, i.e. that to a $99 \%$ confidence, at least $90 \%$ of the population is within the interval. The constant $k$ is defined as

$$
k=\sqrt{\frac{\nu\left(1+\frac{1}{N}\right) z_{(1-p) / 2}^{2}}{\chi_{1-\gamma, \nu}^{2}}},
$$

where $\chi_{1-\gamma, \nu}^{2}$ is the critical value of the chi-square distribution with degrees of freedom $\nu$ that is exceeded with probability $\gamma$, and $z_{(1-p) / 2}^{2}$ is the critical value of the normal distribution associated with cumulative probability $(1-p) / 2$.

Taking the 697 samples from the trajectories, we find

$$
\begin{gathered}
\mu=-0.0083, \\
s=0.371, \\
k=1.546 .
\end{gathered}
$$

Therefore, from Eqs. (1) and (2) we find the upper and lower

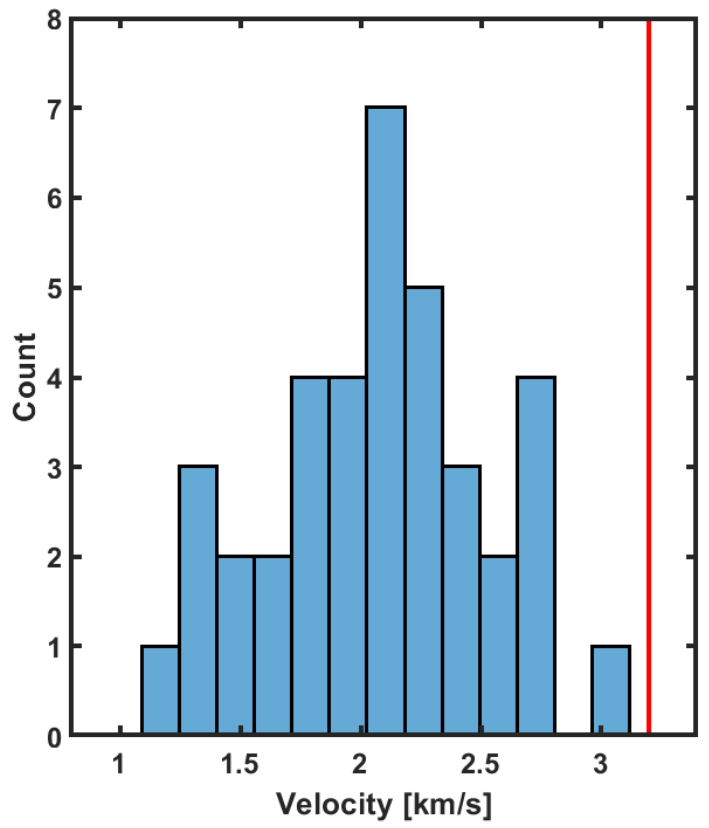

Figure 10. Velocity distribution of the fragments tracked with the presented algorithm. Vertical red line marks the impact velocity.

tolerances,

$$
\begin{gathered}
X_{U}=0.565 \mathrm{~mm} \\
X_{L}=-0.582 \mathrm{~mm} .
\end{gathered}
$$

The upper and lower tolerance intervals are marked with red lines in Fig. 11.

The fragment velocity accuracy can be derived from the position accuracy. If we assume the timing error from the high-speed cameras to be negligible compared to the location error of the fragments then,

$$
v+\epsilon_{v}=\frac{x+\epsilon_{x}}{t+\epsilon t}=v+\frac{\epsilon_{x}}{t},
$$

where $v$ is the velocity, $x$ the position, and $\epsilon$ the respective error. Therefore, the error in the velocity can be calculated as the location error divided by the time,

$$
\epsilon_{v}=\frac{\epsilon_{x}}{t} .
$$

In this experiment, the shortest trajectory was ten frames. Since the timestep between frames was $0.6 \mu \mathrm{s}$, we can estimate the largest tolerance interval for the velocity measurement to be

$$
\epsilon_{v}= \pm \frac{0.582 \mathrm{~mm}}{6 \mu \mathrm{s}}= \pm 96.9 \mathrm{~m} / \mathrm{s} .
$$

This represent a 3 to $8 \%$ error in the velocity, depending on the velocity of the fragment. A more rigorous and thorough accuracy analysis to directly measure systematic errors due to calibration is planned for future setups.

\section{CONCLUSION}

In this paper we have described a trajectory based 3D particle tracking technique used to measure detailed data on individual 


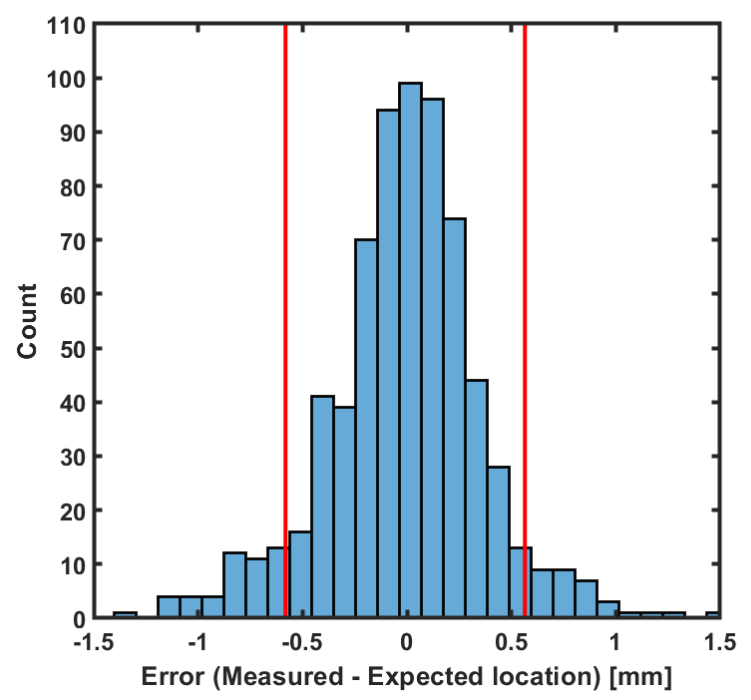

Figure 11. Position error of all tracked fragments. Red lines indicate the tolerance interval.

fragments resulting from HVI. We have shown this method's ability to robustly extract 3D position and time information, allowing properties such as velocity, fragmentation severity, and debris spread to be quantified. Such data is useful for developing a better understanding of HVI phenomena in general, and could be directly put to use in improving spacecraft breakup and space debris environment models.

We plan to continue the development of this method for HVI experiments by measuring fragment sizes and estimating their masses. Having a measure of fragment mass, would not only be valuable new data for space debris models, but would also provide an additional means of verifying the accuracies of the measurements via momentum calculations. Further testing will also be performed to determine the method's performance under the more demanding conditions of higher impact velocities where fragments will be more numerous, smaller, and faster.

\section{REFERENCES}

Fischler, M. a. and Bolles, R. C., 1981. Random Sample Consensus: A Paradigm for Model Fitting with Applicatlons to Image Analysis and Automated Cartography. In: Commun. ACM, Vol. 24, pp. $381-395$.

Gulde, M., Kortmann, L., Ebert, M., Watson, E., Wilk, J. and Schäfer, F., 2017. Robust optical tracking of individual ejecta particles in hypervelocity impact experiments. Meteorit. Planet. Sci. 9, pp. 1-9.

Heineck, J. T., Schultz, P. H. and Anderson, J. L. B., 2002. Application of Three-Component PIV to the Measurement of Hypervelocity Impact Ejecta. J. Vis. 5(3), pp. 233-241.

Hermalyn, B. and Schultz, P. H., 2011. Time-resolved studies of hypervelocity vertical impacts into porous particulate targets: Effects of projectile density on early-time coupling and crater growth. Icarus 216(1), pp. 269-279.

Hermalyn, B., Heineck, J. T., Schairer, E. T. and Peter, H., 2014. Measurement of Ejecta from Hypervelocity Impacts with a Generalized High-Speed Two-Frame 3D Hybrid Particle Tracking Velocimetry Method. In: 17th Int. Symp. Appl. Laser Tech. to Fluid Mech
Higashide, M., Kusano, T., Takayanagi, Y., Arai, K. and Hasegawa, S., 2015. Comparison of Aluminum Alloy and CFRP Bumpers for Space Debris Protection. Procedia Eng. 103, pp. 189-196.

Klinkrad, H., Bendisch, J., Bunte, K. D., Krag, H., Sdunnus, H. and Wegener, P., 2001. The MASTER-99 space debris and meteoroid environment model. Adv. Sp. Res. 28(9), pp. 1355-1366.

Krisko, P. H., 2010. The New NASA Orbital Debris Engineering Model ORDEM 3.0. Technical report.

Krisko, P. H., Flegel, S., Matney, M. J., Jarkey, D. R. and Braun, V., 2015. ORDEM 3.0 and MASTER-2009 modeled debris population comparison. Acta Astronaut. 113, pp. 204-211.

Liou, J. C., 2006. Collision activities in the future orbital debris environment. Adv. Sp. Res. 38(9), pp. 2102-2106.

Maas, H. G., Gruen, a. and Papantoniou, D., 1993. Particle tracking velocimetry in 3-dimensional flows. 1. Photogrammetric determination of partacle coordinates. Exp. Fluids 15(2), pp. 133146.

Nishida, M., Kato, H., Hayashi, K. and Higashide, M., 2013. Ejecta size distribution resulting from hypervelocity impact of spherical projectiles on CFRP laminates. Procedia Eng. 58, pp. 533-542.

Ouellette, N. T., Xu, H. and Bodenschatz, E., 2006. A quantitative study of three-dimensional Lagrangian particle tracking algorithms. Exp. Fluids 40(2), pp. 301-313.

Piekutowski, A. J., 1993. Characteristics of debris clouds produced by hypervelocity impact of aluminum spheres with thin aluminum plates. Int. J. Impact Eng. 14(1-4), pp. 573-586.

Piekutowski, A. J., 1997. Effects of scale on debris cloud properties. Int. J. Impact Eng. 20(6-10), pp. 639-650.

Piekutowski, A. J., 2003. Fragmentation-initiation threshold for spheres impacting at hypervelocity.

Putzar, R. and Schaefer, F., 2009. Experimental Space Debris Simulation At EMI's Calibre 4 MM Two-Stage Light Gas Gun. In: Sp. Debris.

Raffel, M., Willert, C. E., Wereley, S. T. and Kompenhans, J., 2007. Particle Image Velocimetry: A Practical Guide. Springer Berlin Heidelberg.

Ruiz, a., Lopez-de Teruel, P. and Garcia-Mateos, G., 2002. A note on principal point estimability. In: Proc. 16 th Int. Conf. Pattern Recognit., pp. 11-15.

Schimmerohn, M., Watson, E., Gulde, M., Kortmann, L. and Sch, F., 2018. Measuring ejecta characteristics and momentum transfer in experimental simulation of kinetic impact. Acta Astronaut.

Swift, H., 1982. Hypervelocity Impact Mechanics. In: Impact Dyn., John Wiley \& Sons, New York, pp. 215-239.

Watson, E., Gulde, M. and Hiermaier, S., 2017. Fragment Tracking in Hypervelocity Impact Experiments. Procedia Eng. 204, pp. 170-177.

Zhang, Q., Chen, Y., Huang, F. and Long, R., 2008. Experimental study on expansion characteristics of debris clouds produced by oblique hypervelocity impact of LY12 aluminum projectiles with thin LY12 aluminum plates. Int. J. Impact Eng. 35(12), pp. 18841891.

Zhengyou Zhang, 1999. Flexible camera calibration by viewing a plane from unknown orientations. Proc. Seventh IEEE Int. Conf. Comput. Vis. (c), pp. 666-673 vol.1.

Zukas, J., Nicholas, T. and Swift, H., 1982. Impact Dynamics. John Wiley \& Sons, New York. 